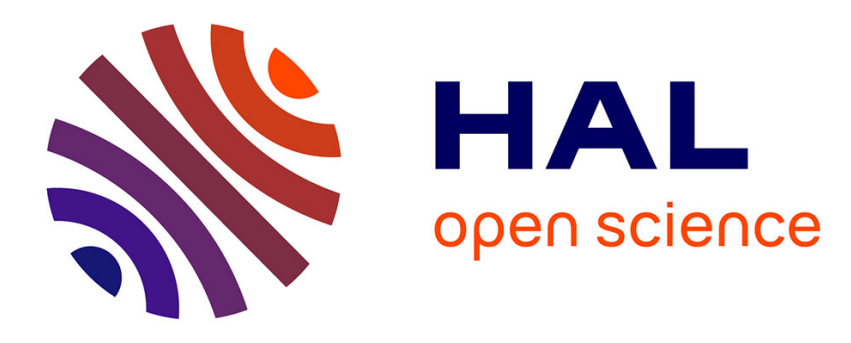

\title{
Mapping of the Marangoni effect in soap films using Young's double-slit experiment
}

Janine Emile, Olivier Emile

\section{To cite this version:}

Janine Emile, Olivier Emile. Mapping of the Marangoni effect in soap films using Young's double-slit experiment. EPL - Europhysics Letters, 2013, 104 (1), pp.14001. 10.1209/0295-5075/104/14001. hal-00881039

\section{HAL Id: hal-00881039 \\ https://hal.science/hal-00881039}

Submitted on 10 Dec 2013

HAL is a multi-disciplinary open access archive for the deposit and dissemination of scientific research documents, whether they are published or not. The documents may come from teaching and research institutions in France or abroad, or from public or private research centers.
L'archive ouverte pluridisciplinaire $\mathbf{H A L}$, est destinée au dépôt et à la diffusion de documents scientifiques de niveau recherche, publiés ou non, émanant des établissements d'enseignement et de recherche français ou étrangers, des laboratoires publics ou privés. 


\title{
Mapping of the Marangoni effect in soap films us- ing Young's double slit experiment.
}

\author{
JANine EMILE ${ }^{1}$ and OLIVIER EMile ${ }^{2}$ \\ 1 IPR, UMR CNRS 6251, Université de Rennes I, 35042 Rennes Cedex, France, U.E. \\ 2 LPL, URU 435, Université de Rennes I, 35042 Rennes Cedex, France, U.E.
}

PACS 47.55.pf - Marangoni convection

PACS $42.87 . \mathrm{Bg}$ - Phase shifting interferometry

PACS 47.20.Bp - Buoyancy-driven instabilities (e.g., Rayleigh-Benard)

\begin{abstract}
We report on the thickness variation measurement of a soap film due to a local perturbation, using Young's double slit experiment configuration. We map a laser-heated deformation of a vertical free-standing draining thin soap film using the differential change of optical path in the interferometer. The experiment has a resolution of about $0.1 \mathrm{~nm}$ and enables to follow the liquid flow dynamics. We evidence a bottleneck formation in the heated region of the film that perturbs the usual flow. Such an experimental set-up could then be adapted to measure other tiny variations in fluctuating hydrodynamics such as capillary waves for example.
\end{abstract}

Introduction. - On the one hand, tears of wine are a superb manifestation of the Marangoni effect [1]. The variation of the surface tension due to the change of alcohol concentration leads to these tears [2-5]. Such an effect can also originate from a temperature gradient at the air/liquid interface and is sometimes called Rayleigh-Bénard convection $[6,7]$ when it is linked to thermal convection. Whereas this effect is often invoked, quantitative measurements are scarce [8-17]. These measurements have been mainly performed, directly using either the naked eye, or with the help of a microscope, or using temperature sensitive markers, or probing the film thickness with interference techniques. On the other hand, tiny length variations in general can be evidenced using optical interferometry measurements $[18$, 19]. Relative variations in the $10^{-17}$ to the $10^{-21}$ range have already been reported [20,21]. Optical interferometry has also been used in the measurement of soap film thicknesses in the $10 \mu \mathrm{m}-200 \mathrm{~nm}$ range [22-24], with a precision of $20 \mathrm{~nm}$. Among all these interferometry techniques, Young's double slit experiment is one of the most popular, fascinating and easy to set up technique. In particular, in quantum mechanics, it has been used to demonstrate the wave particle duality [25] and to evidence phase variations, like in Aharonov-Bohm type experiments [26]. R. Feynman himself said from this experiment: "It is a phenomenon which is impossible to explain in any classical way and which has in it the heart of quantum mechanics" [27]. Back in the optical domain, due to its high simplicity and versatility, one may thus wonder whether Young's double slit experiment could be implemented to measure the thickness variation of soap films via the change of the phase due to the modification of the effective optical length. The aim of this letter is then to investigate potentiality of the double slit experiment by probing the dynamics of the Marangoni effect on a soap film locally heated by a laser. In particular, we will try to map its thickness variations with a 


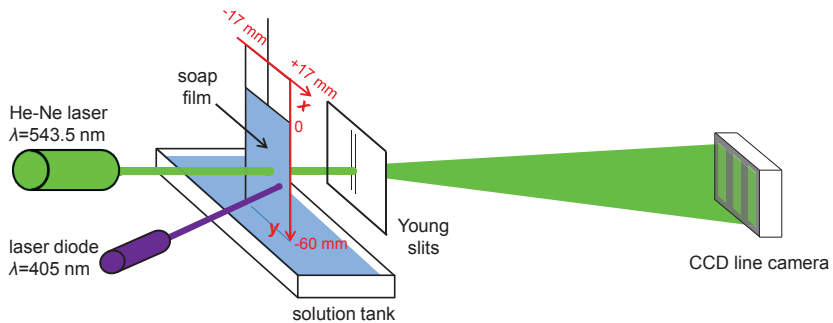

Fig. 1: Experimental set up. A vertical thin soap film is locally heated with a blue laser diode. The differential thickness variation is measured with the interference pattern of a green He-Ne laser in a double slit experiment configuration. The pattern is registered on a CCD line camera connected to a computer.

fraction of a nanometer resolution using Young's double slit experiment.

Experiment. - The soap film is drawn on a rectangular glass frame with a $60 \mathrm{~mm}$ height and a $34 \mathrm{~mm}$ width (see Fig. 1). The vertical film is pull gently upward from a tank containing the foaming solution with a velocity lower than $5 \mathrm{~cm} / \mathrm{s}$. The freestanding film then starts to drain $[29,30]$. The foaming solution consists of $5.4 \%$ sodium lauryldioxyethylene sulphate (SLES, $55.6 \mathrm{~g} / \mathrm{L}$, Cognis) diluted in pure water. To ensure the stability of the film, the surfactant concentration is high, well above the critical micellar concentration and the experiments are performed at a controlled temperature $\mathrm{T}=20.0 \pm 0.5^{\circ} \mathrm{C}$.

The double slit interference pattern is obtained from a green He-Ne laser (Melles Griot, $\lambda=543.5 \mathrm{~nm}, P=5 \mathrm{~mW}$, beam waist size $w=400 \mu \mathrm{m})$. The distance between the two slits is $d=200 \mu \mathrm{m}$. The pattern is observed on a screen located at a distance $L=6 \mathrm{~m}$ from the slits, or registered on a CCD line camera (Thorlabs, LC100), connected to a computer. The interference between the two paths leads to an interference pattern in the $x$ direction that writes $[27]$

$$
I(x) \sim \cos ^{2}\left(\frac{\pi d x}{\lambda L}+\delta \phi\right)
$$

where $I(x)$ is the light intensity on the screen and $\delta \phi$ is the phase difference between the two paths at the slits location. Since the double slit is located just after the soap film (see Fig. 2), this phase difference is related to the differential thickness of the film corresponding to the two paths. A shift of the interference pattern $\Delta x$ (see Fig. 2), then corresponds to a difference between the two optical paths $n\left(e_{1}-e_{2}\right)$, due to the thickness variation

$$
\left(e_{1}-e_{2}\right)=-d \Delta x / n L
$$

where $n \approx 1.33$ is the index of refraction of the film. Unlike X-rays, the use of a visible laser source does not allow to detect different refractive indices related to a multilayer organization of the film, for layer thickness below $20 \mathrm{~nm}$. Our CCD line camera has a resolution of $14 \mu \mathrm{m}$, then the resolution on the differential thickness of the film equals 0.35 $\mathrm{nm}$. A position sensitive detector would have lead to a resolution in the picometer range, or even below.

The film is heated with a focalized diode laser (Dida concept, $\lambda=405 \mathrm{~nm}, P=5 \mathrm{~mW}$, beam waist size $w=300 \mu \mathrm{m}$ ), see Fig. 1 . The beam spot is located just at the middle of the soap film, at the position $x=0 \mathrm{~mm}$ and $y=-30 \mathrm{~mm}$. For a given film thickness, we estimate the power absorbed by the film by measuring the transmitted light through the film. We assess the film thickness independently by an interferometric technique we have already developed [24]. For a $1 \mu \mathrm{m}$ thick film, we measured the transmitted violet light. This wavelength is partially absorbed by the solution contrarily to the green laser used to probe the film thickness. Taking into account the reflection coefficients and the multiple internal 


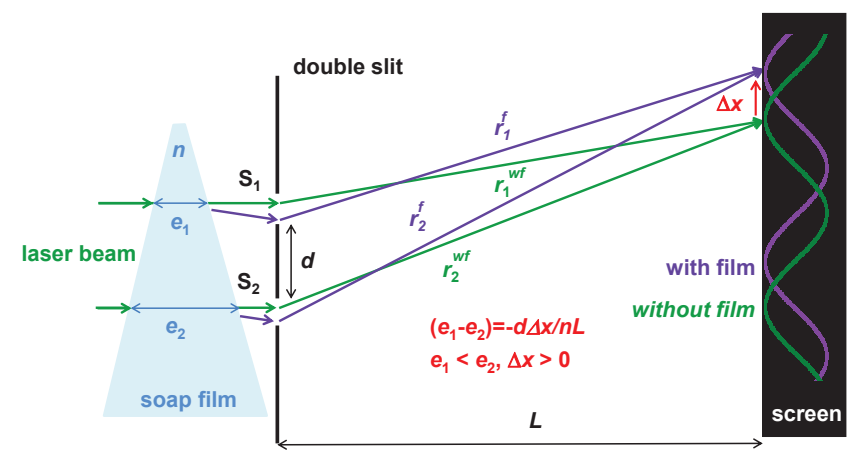

Fig. 2: Principle of the measurement. The change of the film thickness between the two optical interfering beams, induces a modification of the effective optical length. It thus changes the phase difference between the two beams. This leads to a shift of the interference fringes giving access to the thickness gradient of the soap film.

reflections, we estimate the absorbed optical power to be $100 \mu \mathrm{W}$ (corresponding to a $2 \%$ absorption) at the beginning of drainage for a thickness of more than $1 \mu \mathrm{m}$. Some authors have evidence the temperature intensity profile at short times, for an infrared focalized laser beam, heating a liquid film confined between two glass plates [13]. Since here, the film is in a free standing geometry, and the low power laser is not strongly focalized, the heating mechanism could be evaluated in terms of Fourier's law [3], leading to a differential temperature of $25 \mathrm{~K}$. The variation of the index of refraction due to temperature is too low to induce neither optical aberrations nor effective variations of the optical pass of the probe beam. We could change the laser power of the heating violet beam, however, a higher laser power breaks the film and for a lower power, the film gradient is too low after $15 \mathrm{~s}$ drainage to make any relevant measurement. Previous experiments [11,16,31-34] were performed under different conditions in order to measure high temperature gradients only. Devices associated with the deposition of a film on a metallic plate or to the free suspension of a film continuously supplied by a solution pump have been designed to increase artificially the film lifetime, which is not the case here.

Results. - We have then moved the green He-Ne laser source together with the double slits, independently from the frame and from the heating laser, in order to map the deformations of the soap film due to the Marangoni effect. The results appear in Fig. 3a for a horizontal scan (in the $x$ direction), for $y=-30 \mathrm{~mm}$, i.e. corresponding to the height of the heating region. Each measurement is made at least three times to ensure reproducibility. The error bars are shown on the figure. The data give very accurate information on the film thickness gradient and thus must be integrated to extract the true thickness (see Fig. $3 \mathrm{~b}$ ). The measurements have been performed $5 \mathrm{~s}, 10 \mathrm{~s}$ and $15 \mathrm{~s}$ after suspending the soap film, the heating laser being always on. The integration time is $1 \mathrm{~s}$ and the film deformation caused by heating diminishes over time. At the end of the drainage, the film has the same thickness distribution with and without heating. When the heating laser is off, the shift of the interference pattern equals zero, there is neither deformation nor Marangoni effect.

As previously mentioned, the corresponding absolute thickness, with or without heating, has been measured independently using a previously developed experiment [24]. This experiment has a resolution of the order of $20 \mathrm{~nm}$, nearly two orders of magnitude higher than the resolution of the double slit experiment. Besides first experiments do not allow to measure low thickness gradients and to probe grey or black films (from $200 \mathrm{~nm}$ to 10 $\mathrm{nm}$ thickness) that are rather studied by X-ray reflectivity [28]. Nevertheless, the technique of [24] has been used to characterize the film thickness in its middle at $x=0 \mathrm{~mm}$, and 

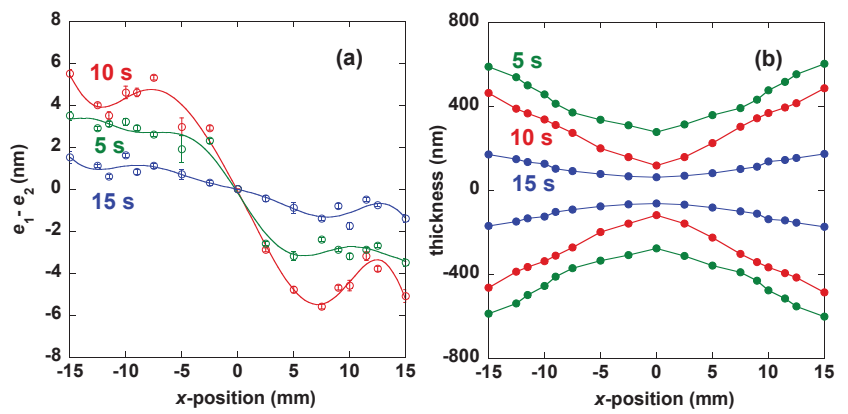

Fig. 3: Horizontal mapping of the soap film. Thickness gradient of the soap film in the horizontal direction $x$ at a height $y=-30 \mathrm{~mm}$ for three different times (5 s-blue, $10 \mathrm{~s}$-red, $15 \mathrm{~s}$-green) and three different thickness. The position $x=0 \mathrm{~mm}, y=-30 \mathrm{~mm}$ corresponds to the zone heated by the blue laser (a). Reconstituted thickness of the soap film from the thickness gradient (b), the solid line is a guide for the eye, thus smoothing the inflections of the curve. Without heating the film thickness is uniform and equals $820 \mathrm{~nm}$ at $5 \mathrm{~s}, 450 \mathrm{~nm}$ at $10 \mathrm{~s}$, and $210 \mathrm{~nm}$ at $15 \mathrm{~s}$. The film is globally thinner when heated.

$y=-30 \mathrm{~mm}$. Then at the other positions, the film thickness has been deduced using the integration procedure the constant being determined at the middle of the film. From the experimental results, it has to be first noticed that the signal is symmetric regarded to the heated region. Second, since the surface tension decreases linearly while increasing the temperature, at small temperature intervals [6], the film thickness mapping is a direct evidence of the heat diffusion in the film [3]. In principle, surface tension gradients could also originate from surfactant concentration, triggered by surface flow. However, due to the structured air/liquid interfaces (above the critical micellar concentration), surface fluctuations are minor. Experimentally, we found that the thermocapillary deformation increases linearly moving away from the heated zone. Besides, whereas the heated zone gets slightly thinner, due to a decrease of the surface tension, the outer zone inflates by nearly a factor of two. The liquid has to by-pass the thinner zone and must thus flow in the lower temperature regions. As we approach the edges of the menisci (about $2 \mathrm{~mm}$ from the frame), this effect is masked by the marginal regeneration caused by a surfactant surplus in the meniscus region [29].

Discussion. - To perform vertical scans, i.e. in the $y$ direction at $x=0 \mathrm{~mm}$ (see Fig. 4a), the slits have been positioned horizontally. We performed the measurement at $t=10 \mathrm{~s}$ but get similar results for the different times. Without heating, the soap film is slightly prismatic; the faces are not parallel to each other $[24,30]$. While heated, it gets thinner at the center, as already noticed with the horizontal scan. This is also confirmed by the direct thickness measurement versus time (see Fig. 4b) at the position where the film is heated. The zone above the heated region inflates upon illumination compared with what is observed without injection, whereas the lower zone gets thinner. The soap film has a more symmetrical shape on both sides of the heating point. The Marangoni effect shades the gravity action. It seems that the lowering of the surface tension due to heating creates a kind of bottleneck that prevents drainage and forces the liquid to circumvent this heated zone. The film gets thinner in the heated region and below, and gets thicker on top of the heated central region as well as on both sides. This is very similar to what has been previously expected or observed for the Marangoni effect but for larger temperature variations and bigger deformations [16,33,34], or even under drying conditions [35, 36].

Curiously, on Fig. 4b, it has to be noticed that at the end of the drainage, the thickness of the film is very similar with and without laser illumination. It turns out that the heating mechanism is not dominant in the dynamics of the thinning any more. Actually, the drainage 

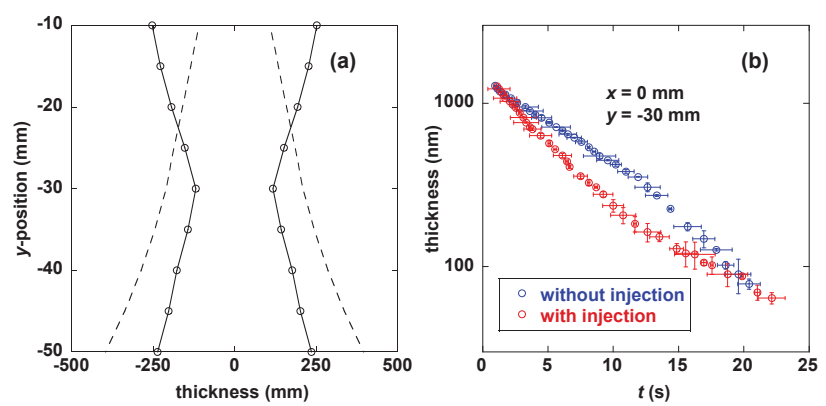

Fig. 4: Vertical mapping of the soap film. Thickness of the thin soap film in the vertical direction $\mathrm{y}$ at a position $x=0$, for $t=10 \mathrm{~s}$. The position $x=0 \mathrm{~mm}, y=-30 \mathrm{~mm}$ corresponds to the zone heated by the blue laser. The thickness variation has been reconstituted from the thickness gradient. The dotted line corresponds to the measured film thickness without heating. It has been measured with the double slit technique, at the same times of draining (a). For other times of drainage, the film has the same vertical shape but the departure from the non heated case is less pronounced. Time evolution of the film thickness at the centre of the film in the heated region (b), with (red curve) and without (blue curve) heating of the film using the direct interferometry technique [24].

of vertical freestanding films in the absence of any perturbation is governed by gravitational, capillary, interfacial forces and thermal convection $[8,12,14,29,30,37]$. In our experimental situation, the film is stratified during drainage, with a well defined organization of micelles in layers parallel to the film plane [24]. The exponential behavior describing the thinning of the soap film is due to the structural forces. Here, after typically $15 \mathrm{~s}$, the liquid is almost ejected and the film reaches a steady state with or without heating (see Fig. 4b). This is also confirmed by the thinning dynamics. Heating only generates solution puncture between the layers of micelles. The evacuation of micelle layers, that should be illustrated by a stepwise thinning, has not been observed in the drainage curve [24]. Actually, the film breaks after $25 \mathrm{~s}$, just before the stepwise thinning is supposed to play a major role [38]. The theoretical description of the profile is not as trivial as it may appear. In the absence of heating, the drainage curve described by an exponential function is mainly due to structural forces needed to stabilize the micelle layers and not by gravitational and capillary forces usually incorporated in hydrodynamic models. In the presence of heating, the solution transfer cannot be described by a simple one-dimensional equation: the liquid is necessarily influenced by gravity and part of the heat is evacuated towards the reservoir. The liquid can either flow in the $y$ direction or along the frame as it is expelled in the $x$ direction toward the meniscus. Thus since the liquid in the film can move towards the frame and then flows along it, there is no mass conservation within the film.

Conclusion. - To conclude, we used the high versatility of Young's double slit experiment to perform a differential mapping of a soap film. By local heating of the film by a laser, we measured the Marangoni effect. We experimentally evidence a bottleneck in the heated zone that force the liquid to circumvent this zone leading to an inflation of the film thickness above this zone. These results are in agreement with previous predictions and observations, but the high sensitivity of the technique allows to probe very tiny deformations far below what has been observed previously, and far before the apparition of convection vortices $[16,33]$. This easy to implement technique shines some new light on the film dynamics in the presence of a local defect and could be applied to investigate [39] or to control [40] interfacial instabilities in thin liquid films. Besides, due to the high precision of the measurement, it is also appropriate for measuring small optical index variation in the case of light guiding in soap films [41] or tiny thermally induced deformations such as capillary 
waves on liquid surfaces that are only studied by X-ray reflectivity [42].

$$
* * *
$$

The authors thank Alexis Keller and Federico Casanova for valuable help, and Jean René Thébault and Alain Faisant for technical assistance.

\section{REFERENCES}

[1] Thomson J., Philos. Mag., 10 (1855) 330.

[2] Gibbs J.W., Trans. Connecticut Acad. Art Sci., 3 (1878) 343.

[3] Chandrasekhar S., Hydrodynamic and hydromagnetic stability (Dover, New York) 1981.

[4] Pearson J.R.A., J. Fluid Mech., 4 (1958) 489.

[5] Fournier J.B. and Cazabat A.M., Europhys. Lett., 20 (1992) 517.

[6] Getling A.V., Rayleigh-Bénard convection: structure and dynamics (World Scientific, New York) 1988.

[7] VanHook S.J., Schatz M.F., McCormick W.D., Swift J.B. and Swinney H.L., Phys. Rev. Lett., 75 (1995) 4397.

[8] Oron A., Davis S.H. and Bankoff S.G., Rev. Mod. Phys., 69 (1997) 931.

[9] Kellay H. and Goldburg W.I., Rep. Prog. Phys., 65 (2002) 845.

[10] Stroock A.D., Ismagilov R.F., Stone H.A. and Whitesides G.M., Langmuir, 19 (2003) 4358.

[11] Zaitsev D.V., Kabov O.A. and Evseev A.R., Exp. Fluids, 34 (2003) 748.

[12] Berg S., Adelizzi E.A. and Troian S.M, Langmuir, 21 (2005) 3867.

[13] Cordero M.L., Verneil E., Gallaire F. and Baroud C.N., Phys. Rev. E, 79 (2009) 011201.

[14] Tran T., Chakraborty P., Guttenberg N., Prescott A., Kellay H., Goldburg W., Goldenfeld N. and Gioia G., Nature Phys., 6 (2010) 438.

[15] Matsuoka H., Oka K., Yamashita Y., Saeki F. and Fukui S., Microsyst. Technol., 17 (2011) 983.

[16] Adami N., Dorbolo S. and Caps H., Phys. Rev E, 84 (2011) 046316.

[17] Sempels W., Dier R.D., Mizuno H., Hofkens J. and Vermant J., Nat. Commun., 4 (2013) 1757.

[18] Wilkins S.W., Gureyev T.E., Gao D., Pogang A. and Syevenson A.W., Nature, 384 (1996) 335.

[19] Oliver W.D., Yu Y., Lee J.C., Bergaren K.K., Levitov L.S. and Orlando T.P., Science, $\mathbf{3 1 0}$ (2005) 1653.

[20] Eisele Ch., Nevsky A.Yu. and Schiller S., Phys. Rev. Lett., 103 (2009) 090401.

[21] Ligo Scientific Collaboration, Nature Phys., 7 (2011) 962.

[22] Ropars G., Chauvat D. Le Floch A., O’Sullivan-Hale M.N. and Boyd R.W., Appl. Phys. Lett., 88 (2006) 234104.

[23] Afanasyev Y.D., Andrews G.T. and Deacon C.G., Am. J. Phys., 79 (2011) 1079.

[24] Emile J., Casanova F., Loas, G. and Emile O., Soft Matter, 8 (2012) 7223.

[25] Bohr N., Atomic physics and human knowledge (Wiley, New York) 1958.

[26] Peskin M. and Tonomura A., The Aharonov-Bohm effect (Springer-Verlag, Berlin) 1989.

[27] Feynman R.P., Leighton R. and Sands M., The Feynman lectures on physics, Vol. 3 (Massachusetts, Addison-Wesley) 1966.

[28] Bélorgey O. and Benattar J.J., Phys. Rev. Lett., 66 (1991) 313.

[29] Mysels K.J., Shinoda K. and Frankel S., Soap films, studies of their thinning (Pergamon, New York) 1959.

[30] Couder Y., Chomaz J.M. and Rabaud M., Physica D, 37 (1989) 384.

[31] Cazabat A.M., Heslot F., Troian S.M. and Carles P., Nature, 346 (1990) 824.

[32] brzoska J.B., Brochart-Wyart F. and Rondelez F., Europhys. Lett., 19 (1992) 97.

[33] Martin B. and Wu X.L., Phys. Rev. Lett., 80 (1998) 1892.

[34] Zhang J., Wu X.L. and Rashidnia N., Phys. Fluids, 18 (2006) 085110.

[35] Chauvet F., Dehaeck S. and Colinet P., EPL, 99 (2012) 34001. 
[36] Doumenc F. and Guerrier B., EPL, 103 (2013) 14001.

[37] Winkler M., Kofod G., Krastev R., Stöckle S. and Abel M., Phys. Rev. Lett., 110 (2013) 094501.

[38] Danov K.D., Basheva E.S., Kralchevsky P.A., Ananthapadmanabhan K.P. and Lips A., Adv. Colloid Interface Sci., 168 (2011) 50.

[39] Schatz M.F. and Neitzel G.P., Annu. Rev. Fluid Mech., 33 (2001) 93.

[40] Al-Housseiny T.T., Tsai P.C.A. and Stone H.A., Nature Phys., 8 (2012) 747.

[41] Emile J., Emile O. and casanova F., EPL., 101 (2013) 34005.

[42] Daillant J. and Alba M., Rep. Prog. Phys., 63 (2000) 1725. 